Dementia and Geriatric Cognitive Disorders
Accepted: May 12, 2008

Published online: July 10, 2008

\title{
Prediction of Institutionalisation in Dementia
}

\section{A Systematic Review}

\author{
Melanie Luppa ${ }^{a}$ Tobias Luck ${ }^{a}$ Elmar Brähler ${ }^{c}$ Hans-Helmut König ${ }^{b}$ \\ Steffi G. Riedel-Heller ${ }^{\mathrm{a}}$ \\ ${ }^{a}$ Department of Psychiatry, Public Mental Health Research Unit, ${ }^{b}$ Department of Psychiatry, \\ Health Economics Research Unit, and 'Department of Medical Psychology and Medical Sociology, \\ University of Leipzig, Leipzig, Germany
}

\section{Key Words}

Institutionalisation in dementia • Nursing home placement • Dementia

\begin{abstract}
Background/Aims: In the past decades, a substantial number of studies considered factors influencing institutionalisation of persons with dementia. This study reviews recent work on predictors of actual institutionalisation in dementia. Method: Relevant articles were identified by a systematic search of the literature. Studies were considered which included persons aged 65 and over, and whose results were based on prospective design and on multivariate statistical analyses. Results: Forty-two studies were identified. The rate of institutionalisation increased from $20 \%$ in the first year after diagnosis to $50 \%$ after 5 years. Median time to institutionalisation was estimated between 30 and 40 months. Predictors of institutionalisation were classified according to a provided conceptual framework in the categories sociodemographic and relationship characteristics of persons with dementia and caregivers, primary stressors, secondary stressors and resources. Conclusion: The overview of research activities in this area showed a lack of methodological strength in a large part of identified studies. Nevertheless, a lot of well-examined and less highlighted predictors could be identified.

Copyright $\odot 2008$ S. Karger AG, Basel
\end{abstract}

\section{Introduction}

Dementia is one of the most common and serious disorders in late life. It is associated with progressive deterioration in cognitive and physical functioning and has profound consequences for the persons suffering from dementia, their family and caregivers. Currently, there are about 24.3 million people with dementia in the world, with 4.6 million new cases of dementia every year. The number of people affected will double every 20 years to 81.1 million by 2040 [1]. In the USA, 8.9 million people care for relatives with dementia [2], and this number is growing [3]. From an economic perspective, the total worldwide societal costs of dementia were estimated to be USD 315.4 billion in 2005 [4]. An essential part of the societal costs (33\%) was for informal care (USD 105 billion) [4], often provided by family members.

Due to the global burden, and especially, due to the burden on informal caregivers, dementia is the main cause for institutionalisation in the elderly [5]. However, most elderly people prefer to live as long as possible in their familiar surroundings [6]. On the one hand, it may be preferable for these patients to remain in their homes, not only for economic reasons, but also because they remain able to maintain the integrity of their social network, preserve environmental landmarks, and enjoy a better quality of life. On the other hand, institutionalisa-

\section{KARGER \\ Fax +4161306 1234 \\ E-Mail karger@karger.ch}

www.karger.com
(C) 2008 S. Karger AG, Basel

$1420-8008 / 08 / 0261-0065 \$ 24.50 / 0$

Accessible online at:

www.karger.com/dem
Melanie Luppa

Department of Psychiatry, Public Mental Health Research Unit, University of Leipzig

Johannisallee 20, DE-04317 Leipzig (Germany)

Tel. +49 341972 4534, Fax +49341972 4539

E-Mail Melanie.Luppa@medizin.uni-leipzig.de 


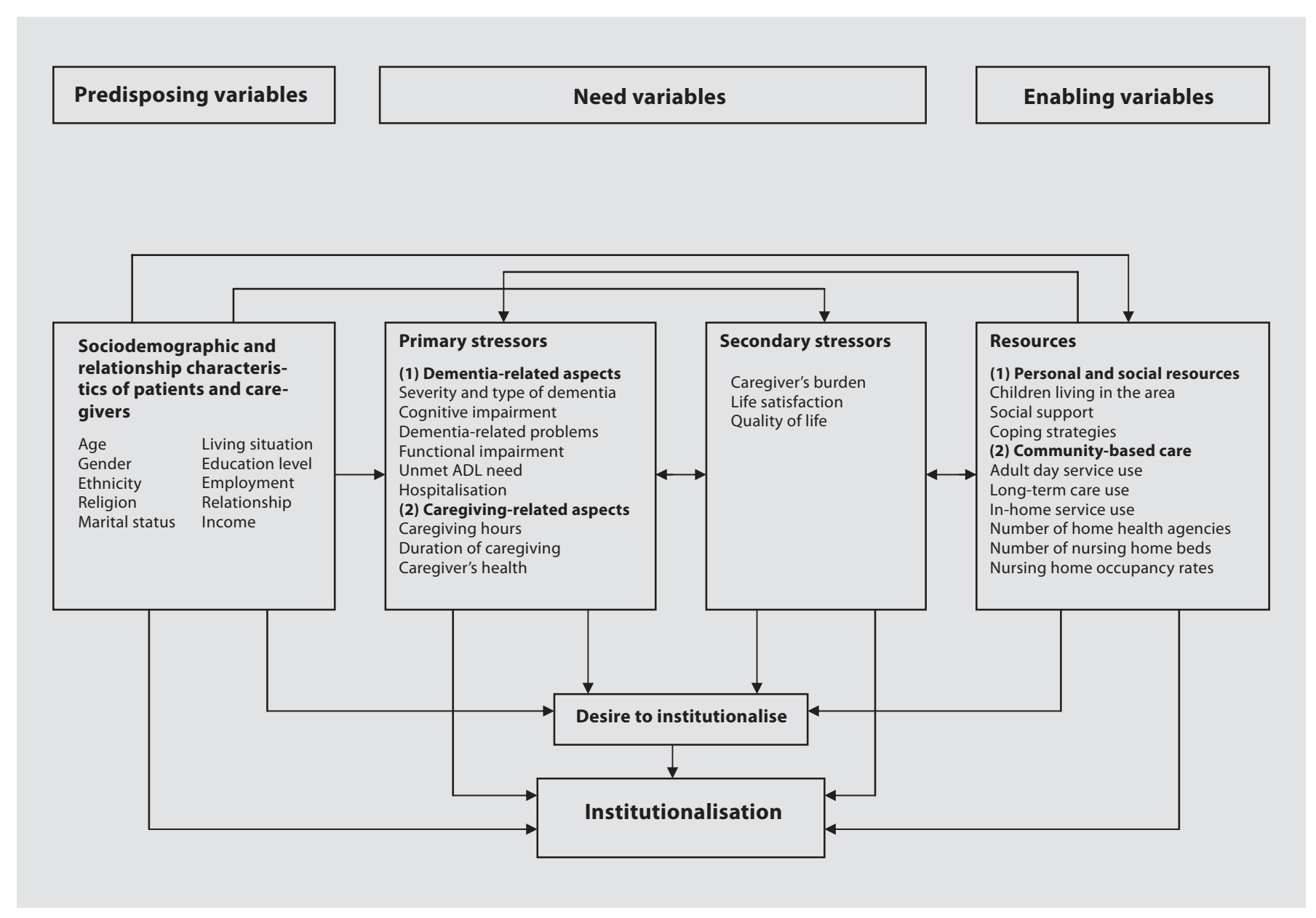

Fig. 1. Conceptual framework of factors influencing institutionalisation. ADL = Activities of daily living.

tion can be expedient for subjects who suffer from more severe dementia, because caring for these patients at home is a stressful and demanding process that affects caregivers' psychological and physical well-being [7] and jeopardizes the feasibility of continued home care. If institutionalisation becomes inevitable, the decision to institutionalise a patient with dementia is complex and involves a number of factors that interact in the decision-making process such as patient and caregiver characteristics as well as contextual factors relating to the care situation (e.g. informal and formal support, physical environment) [8]. The identification of those factors associated with nursing home placement (NHP) facilitated the application of health and social strategies to permit those patients with dementia to stay in their homes as long as possible and to assure that steps for a well-timed institutionalisation were initiated.
In the past 30 years, a substantial number of studies considered factors associated with long-term institutionalisation and NHP of persons with dementia, partially with contrary results. In the international literature, a review of institutionalisation of persons with dementia is not found. This is the first article which systematically reviews all published studies analysing prediction of actual institutionalisation in dementia with the following objectives: (1) describing methodological characteristics of studies, (2) analysing the rates of and time until institutionalisation in persons with dementia, (3) considering predictors of risk for and time to institutionalisation, (4) subsuming predictors in the following conceptual framework and (5) summarising current research findings and drawing conclusions for future research in this area. 


\section{Conceptual Framework}

In view of structuring the results of the review, we aimed to provide a conceptual framework for predicting the actual institutionalisation by combining the behavioural model of health service use $[9,10]$ with the stress process model for dementia caregiving of Pearlin et al. [11] and the conceptual framework for predicting institutionalisation by Pruchno et al. [12] (fig. 1). The behavioural model of Andersen $[9,10]$ suggests that people's use of health services and NHP is a function of predisposing, need and enabling characteristics of the individual. Predisposing variables were demographic factors, social structure characteristics and health beliefs. Collectively, they represent the sociocultural element of the behavioural model. We considered the sociodemographic and relationship characteristics of the care recipient and the caregiver (as described by Pearlin et al. [11] and Pruchno et al. [12]) as predisposing variables for institutionalisation in dementia. The need variables on the one hand included the amount of illness that an individual (also caregiver) perceives, and on the other hand represent professionally evaluated need. We subsumed primary and secondary stressors associated with dementia according to the stress process model $[11,12]$ under this term. Primary stressors stem directly from the needs of the patients and the nature and magnitude of the care demanded by these needs [11]. Behavioural and psychological symptoms of dementia (BPSD) as well as characteristics of the caregiving situation were included in primary stressors. The secondary stressors include the caregiver's appraisal of the burden or stress associated with the caregiving situation [12], or, in other words, stress caused by the role or intrapsychic strains of the caregiver [11]. The enabling component contains factors which make health services and NHP available to the individual for use and should include familial and community resources $[9,10]$. Following Pruchno et al. [12], we assigned personal and social resources as well as community-based care supporting the caregivers in their caregiving tasks to the enabling domain.

It can be expected that these described sets of variables contribute to the prediction of both the desire to institutionalise a person with dementia as well as actual institutionalisation. We focused solely on prediction of actual institutionalisation.

\section{Methods}

A systematic literature search was conducted. Relevant publications on institutionalisation in dementia were identified by searching the electronic databases MEDLINE, Web of Science,
Cochrane Library and PSYNDEXplus as well as bibliographies of identified articles. 'Institutionalisation', 'nursing home placement', 'nursing home admission' and 'dementia' served as keywords. The following selection criteria were used: (1) studies with a prospective design, (2) studies identifying influencing factors by the use of multivariate analyses (for adjustment of covariates), (3) studies with the majority of respondents being 65 years and older, (4) studies focusing on factors of actual institutionalisation (instead of desire or intention to institutionalise).

\section{Results}

The systematic literature search identified 509 potentially relevant articles ${ }^{1}$ of which 416 were excluded based on their title and abstract. Nine articles which were found in reference lists of the identified articles were added to the remaining 93 articles. However, after perusing the full articles, 51 were rejected. Forty-two studies fulfilled the selection criteria and were assessed and subjected to the detailed analysis. Table 1 provides an overview of the included 42 studies sorted by publication year. Two thirds of the studies were conducted in the USA, most others in Europe, 1 in Australia and 1 in Canada. The studies showed a wide range of sampling designs. Most of the studies recruited samples from Alzheimer's disease (AD) diagnostic centres or memory clinics (MC) as well as samples of patients receiving community-based long-term care (CLTC). Others analysed data files of health care providers, and three studies were related to population-based samples [14-16]. Half of the studies included samples of patients and their caregivers, others exclusively included patients or caregivers, and a few obtained information on the patients from the caregivers (table 1). Diagnosis of dementia was based in half of the studies on criteria of international classification systems or on NINCDS-ADRDA criteria including either various types of dementia $(n=11)$ or solely $A D(n=$ 10). Fourteen studies included persons with dementia according to physician's diagnosis without information about diagnostic criteria. The remaining studies reported no further information on diagnostic criteria.

\section{Institutionalisation in Dementia}

Admission rates to long-term institutions of community-dwelling elderly were reported by most of the stud-

1 All of the potentially relevant articles were evaluated by two reviewers (M.L. and T.L.). Studies were evaluated according to a study appraisal system used by the UK National Institute for Health and Clinical Excellence (NICE) [13]. This system assigns a grade of either '++', '+' or '-' to each study. Studies that were graded as '-' were excluded. There was no case of disagreement between the reviewers on the exclusion of studies. 
Table 1. Studies of institutionalisation of persons with dementia

\begin{tabular}{|c|c|c|c|c|c|c|c|c|c|c|c|c|c|}
\hline $\begin{array}{l}\text { Study; } \\
\text { year; } \\
\text { country }\end{array}$ & $\begin{array}{c}\mathrm{n}^{1} \mid \mathrm{n}^{2} \\
\mathrm{n}^{3}\end{array}$ & $\mathrm{~A}^{4}$ & $\begin{array}{l}\mathrm{OI} / \\
\mathrm{TP}^{5}\end{array}$ & $\begin{array}{l}\text { DS; } \\
D^{6}\end{array}$ & $\mathrm{IR}^{7}$ & $\begin{array}{l}\text { Time } \\
\text { and/or } \\
\text { risk }^{8}\end{array}$ & $\begin{array}{l}\text { CR factors }{ }^{9} \\
\left(I^{10}\right)\end{array}$ & Risk $^{11}$ & $\begin{array}{l}{ }^{1} \text { Risk } \\
\text { type }\end{array}$ & $\begin{array}{l}\text { CG factors } 9 \\
\left(\mathrm{I}^{10}\right)\end{array}$ & Risk $^{11}$ & $\begin{array}{l}\text { Risk } \\
\text { type }\end{array}$ & $\begin{array}{l}\text { Methodical } \\
\text { notes }^{12}\end{array}$ \\
\hline $\begin{array}{l}\text { Andel et al. } \\
{[21] ; 2007 \text {; USA }}\end{array}$ & 587 & $\begin{array}{l}65+ \\
79\end{array}$ & $\begin{array}{l}4 / \\
\mathrm{n} / \mathrm{a}\end{array}$ & $\begin{array}{l}\text { p.d.; } \\
\text { dementia }\end{array}$ & $\begin{array}{l}55 \%(4) \\
14 \text { months } \\
\text { (entry) }\end{array}$ & risk & $\begin{array}{l}\text { Age, }>85 \text { years } \\
\text { Ethnicity, white American } \\
\text { Marital status (ref. married), } \\
\text { widowed }\end{array}$ & $\begin{array}{l}1.48 \\
1.69 \\
0.72\end{array}$ & $\mathrm{HR}$ & & & & $\begin{array}{l}\text { Data files of } \\
\text { outpatients; } \\
\text { CPHR }\end{array}$ \\
\hline $\begin{array}{l}\text { Coehlo et al. } \\
\text { [35]; 2007; USA }\end{array}$ & 92 & 72 & $0.5 / 2$ & $\begin{array}{l}\mathrm{n} / \mathrm{a} ; \\
\text { dementia }\end{array}$ & $\mathrm{n} / \mathrm{a}$ & $\begin{array}{l}\text { time } \\
\text { risk }\end{array}$ & $\begin{array}{l}\text { Duration of dementia, } \\
>4 \text { years } \\
\text { BPSD } \uparrow(\mathrm{NPI}) \\
\text { Functional impairment } \uparrow \\
(\text { ADL) }\end{array}$ & $\begin{array}{l}\uparrow \\
\uparrow \\
\uparrow\end{array}$ & & $\begin{array}{l}\text { Depression, } \\
\text { presence (CES-D) } \\
\text { Employment status, } \\
\text { employed }\end{array}$ & $\uparrow$ & & $\begin{array}{l}\text { AD-centre; } \\
\text { CPHR }\end{array}$ \\
\hline $\begin{array}{l}\text { Luck et al. } \\
\text { [14]; 2007; } \\
\text { Germany }\end{array}$ & 109 & $\begin{array}{l}75+ \\
86\end{array}$ & $1.4 / 6$ & $\begin{array}{l}\text { DSM-IV; } \\
\text { AD, VD, } \\
\text { OD }\end{array}$ & $\begin{array}{l}48 \%(6) \\
35 \text { months } \\
\text { (onset) }\end{array}$ & time & $\begin{array}{l}\text { Marital status } \\
\text { (ref. married), } \\
\text { widowed/divorced }\end{array}$ & 4.50 & $\mathrm{HR}$ & & & & $\begin{array}{l}\text { Population- } \\
\text { based } \\
\text { sample } \\
\text { (incident } \\
\text { cases); } \\
\text { CPHR }\end{array}$ \\
\hline $\begin{array}{l}\text { Buhr et al. [39]; } \\
\text { 2006; USA } \\
\text { (NLCS) }\end{array}$ & 2,200 & $60+$ & $3 / 4$ & $\begin{array}{l}\text { ICD-9 } \\
\text { AD, VD }\end{array}$ & $26 \%(3)$ & risk & $\begin{array}{l}\text { BPSD } \uparrow(B R S-D) \\
\text { Psychotic symptoms, } \\
\text { presence (BRS-D) }\end{array}$ & $\begin{array}{l}1.07 \\
1.06\end{array}$ & HR & $\begin{array}{l}\text { Ethnicity, } \\
\text { white American } \\
\text { Life satisfaction } \downarrow \\
\text { Need for skilled } \\
\text { care } \uparrow\end{array}$ & $\begin{array}{l}1.74 \\
1.52 \\
1.08\end{array}$ & $\mathrm{HR}$ & $\begin{array}{l}\text { Data files of } \\
\text { outpatients; } \\
\text { CPHR }\end{array}$ \\
\hline $\begin{array}{l}\text { Gaugler et al. } \\
\text { [36]; 2006; USA } \\
\text { (MADDE) }\end{array}$ & $\begin{array}{c}324 \\
\text { (Latinos) }\end{array}$ & 78 & $3 / 6$ & $\begin{array}{l}\text { p.d.; } \\
\text { dementia }\end{array}$ & $23 \%(3)$ & risk & Living situation, with CG & 0.28 & $\mathrm{HR}$ & $\begin{array}{l}\text { Depression, presence } \\
\text { (GDS) } \\
\text { Income } \uparrow \\
\text { CG hours } \uparrow \\
\text { ADS use } \uparrow\end{array}$ & $\begin{array}{l}1.18 \\
1.15 \\
1.01 \\
1.01\end{array}$ & HR & $\begin{array}{l}\text { CLTC user; } \\
\text { CPHR }\end{array}$ \\
\hline $\begin{array}{l}\text { Soto et al. } \\
{[59] ; 2006 ;} \\
\text { France }\end{array}$ & 585 & $65-85$ & $1 / 2$ & $\begin{array}{l}\text { p.d.; } \\
\text { AD }\end{array}$ & $\mathrm{n} / \mathrm{a}$ & risk & $\begin{array}{l}\text { Living situation (ref. with } \\
\text { spouse), } \\
\text { with family } \\
\text { alone }\end{array}$ & $\begin{array}{l}3.30 \\
4.33\end{array}$ & OR & $\begin{array}{l}\text { Burden } \uparrow \text {, } \\
(\mathrm{ZBI}>20) \\
(\mathrm{ZBI}>40)\end{array}$ & $\begin{array}{l}1.21 \\
3.33\end{array}$ & OR & $\begin{array}{l}\text { AD-centre; } \\
\text { LR }\end{array}$ \\
\hline $\begin{array}{l}\text { Argimon et al. } \\
\text { [43]; 2005; } \\
\text { Spain }\end{array}$ & 181 & 79 & $1 / 3$ & $\begin{array}{l}\text { p.d. } \\
\text { AD, VD, } \\
\text { MD }\end{array}$ & $11 \%(1)$ & risk & & & & $\begin{array}{l}\text { Health-related } \\
\text { QoL } \downarrow \text { (SF-36) } \\
\text { (subtests: level of } \\
\text { physical function, } \\
\text { general health } \\
\text { and physical role) }\end{array}$ & 6.40 & OR & $\begin{array}{l}\text { Primary } \\
\text { health } \\
\text { centre; } \\
\text { LR }\end{array}$ \\
\hline $\begin{array}{l}\text { De Vugt et al. } \\
\text { [32]; 2005; The } \\
\text { Netherlands } \\
\text { (MAASBED) }\end{array}$ & 119 & 79 & $2 / 4$ & $\begin{array}{l}\text { DSM-IV; } \\
\text { AD, VD, } \\
\text { FTD, OD }\end{array}$ & $\begin{array}{l}13 \%(0.5) \\
23 \%(1) \\
34 \%(1.5) \\
41 \%(2)\end{array}$ & time & $\begin{array}{l}\text { CG relationship } \\
\text { (ref. spouse), child }\end{array}$ & 3.10 & $\mathrm{HR}$ & $\begin{array}{l}\text { Distress with } \\
\text { BPSD of CR } \uparrow \\
\text { (NPI) }\end{array}$ & 2.70 & $\mathrm{HR}$ & $\begin{array}{l}\text { Memory } \\
\text { clinic; } \\
\text { CPHR }\end{array}$ \\
\hline $\begin{array}{l}\text { Dorenlot et al. } \\
{[22] ; 2005 ;} \\
\text { France } \\
\text { (DEPAD } \\
\text { study) }\end{array}$ & 348 & 81 & $1 / 3$ & $\begin{array}{l}\text { DSM-IV; } \\
\text { NINCDS- } \\
\text { ADRDA } \\
\text { AD, VD, } \\
\text { MD, FTD, } \\
\text { OD, LTD }\end{array}$ & $33 \%(1)$ & risk & $\begin{array}{l}\text { Age } \uparrow \\
\text { Living situation, alone } \\
\text { Severity of dementia } \uparrow \\
\text { (CDR) } \\
\text { Cognitive impairment } \uparrow \\
\text { (MMSE score }<17 \text { ) } \\
\text { Functional impairment } \uparrow \\
\text { (IADL) } \\
\text { Major depression, presence } \\
\text { (MDS-HC) }\end{array}$ & $\begin{array}{l}2.00 \\
2.00 \\
1.80 \\
\\
1.80 \\
1.30 \\
1.60\end{array}$ & $\mathrm{HR}$ & & & & $\begin{array}{l}\text { Memory } \\
\text { clinic; } \\
\text { CPHR }\end{array}$ \\
\hline $\begin{array}{l}\text { Gaugler et al. } \\
{[31,44,60]} \\
\text { 2005; USA } \\
\text { (MADDE) }\end{array}$ & 5,831 & 79 & $1.5 / 4$ & $\begin{array}{l}\text { p.d.; } \\
\text { dementia }\end{array}$ & $\begin{array}{l}12 \%(0.5) \\
24 \%(1) \\
36 \%(1.5)\end{array}$ & Risk & $\begin{array}{l}\text { Age } \uparrow \\
\text { Gender, female } \\
\text { Ethnicity, white American } \\
\text { Living situation, with CG } \\
\text { Functional impair- } \\
\text { ment } \downarrow \text { (ADL/IADL) } \\
\text { BPSD } \uparrow(\mathrm{MBPC})\end{array}$ & $\begin{array}{l}1.01 \\
0.81 \\
1.92 \\
0.79 \\
\\
0.69 \\
1.05\end{array}$ & OR & $\begin{array}{l}\text { Age } \uparrow \\
\text { Gender, female } \\
\text { Income } \uparrow \\
\text { Dependencies in } \\
\text { IADL } \uparrow \\
\text { Unmet ADL need } \uparrow\end{array}$ & $\begin{array}{l}1.01 \\
0.78 \\
1.02 \\
\\
1.11 \\
1.77\end{array}$ & OR & $\begin{array}{l}\text { CLTC user; } \\
\text { CPHR }\end{array}$ \\
\hline
\end{tabular}


Table 1 (continued)

\begin{tabular}{|c|c|c|c|c|c|c|c|c|c|c|c|c|c|}
\hline $\begin{array}{l}\text { Study; } \\
\text { year; } \\
\text { country }\end{array}$ & $\begin{array}{c}\mathrm{n}^{1} \mid \mathrm{n}^{2} \\
\mathrm{n}^{3}\end{array}$ & $\mathrm{~A}^{4}$ & $\begin{array}{l}\mathrm{OI} / \\
\mathrm{TP}^{5}\end{array}$ & $\begin{array}{l}\text { DS; } \\
D^{6}\end{array}$ & $\mathrm{IR}^{7}$ & $\begin{array}{l}\text { Time } \\
\text { and/or } \\
\text { risk }^{8}\end{array}$ & $\begin{array}{l}\text { CR factors } 9 \\
\left(I^{10}\right)\end{array}$ & Risk $^{11}$ & $\begin{array}{l}{ }^{1} \text { Risk } \\
\text { type }\end{array}$ & $\begin{array}{l}\text { CG factors } 9 \\
\left(\mathrm{I}^{10}\right)\end{array}$ & Risk $^{11}$ & $\begin{array}{l}\text { Risk } \\
\text { type }\end{array}$ & $\begin{array}{l}\text { Methodical } \\
\text { notes }^{12}\end{array}$ \\
\hline & 4,761 & & $3 / 7$ & & $46 \%(3)$ & risk & $\begin{array}{l}\text { Cognitive impairment } \uparrow \\
\text { (MMSE) }\end{array}$ & $\uparrow$ & & $\begin{array}{l}\text { Use of in-home help } \\
\text { services, earlier } \\
\text { Burden } \uparrow(\mathrm{ZBI}) \\
\text { ADS use } \uparrow \\
\text { Duration as CG } \downarrow\end{array}$ & $\begin{array}{l}0.83 \\
\uparrow \\
\uparrow \\
\uparrow\end{array}$ & HR & \\
\hline $\begin{array}{l}\text { McCann et al. } \\
\text { [23]; 2005; USA }\end{array}$ & 516 & $65+$ & $4 / 9$ & $\begin{array}{l}\text { NINCDS- } \\
\text { ADRDA; } \\
\text { AD }\end{array}$ & $35 \%(4)$ & time & $\begin{array}{l}\text { Age } \uparrow \\
\text { Gender, male } \\
\text { Ethnicity, AA } \\
\text { Cognitive impairment } \downarrow \\
\text { (MMSE) } \\
\text { Functional impairment } \uparrow \\
\text { (RBDS) } \\
\text { Hospitalisation in } \\
\text { previous } 3 \text { months }\end{array}$ & $\begin{array}{l}1.01 \\
1.45 \\
0.68 \\
0.98 \\
1.32 \\
1.54\end{array}$ & $\mathrm{HR}$ & $\begin{array}{l}\text { Age } \uparrow \\
\text { Burden } \uparrow \\
\text { ADS use } \uparrow\end{array}$ & $\begin{array}{l}1.01 \\
1.05 \\
1.33\end{array}$ & HR & $\begin{array}{l}\text { ADS user, } \\
\text { AD-centre; } \\
\text { CPHR }\end{array}$ \\
\hline $\begin{array}{l}\text { Gaugler et al. } \\
\text { [38]; 2004; USA } \\
\text { (MADDE) }\end{array}$ & $\begin{array}{l}667 \\
(\mathrm{AA})\end{array}$ & $50+$ & $3 / 7$ & $\begin{array}{l}\text { p.d.; } \\
\text { dementia }\end{array}$ & $30 \%(3)$ & risk & $\begin{array}{l}\text { Age } \downarrow \\
\text { Gender, male } \\
\text { Cognitive impairment } \uparrow \\
\text { (MMSE score }<11 \text { ) }\end{array}$ & $\begin{array}{l}0.44 \\
1.62 \\
4.59\end{array}$ & HR & $\begin{array}{l}\text { Burden } \uparrow \\
(\mathrm{ZBI}>18)\end{array}$ & 2.47 & $\mathrm{HR}$ & $\begin{array}{l}\text { CLTC user; } \\
\text { CPHR }\end{array}$ \\
\hline $\begin{array}{l}\text { Gilley et al. } \\
\text { [28]; 2004; USA }\end{array}$ & 410 & $\begin{array}{l}45-95 \\
76\end{array}$ & $5 / 6$ & $\begin{array}{l}\text { NINCDS- } \\
\text { ADRDA; } \\
\text { AD }\end{array}$ & $\begin{array}{l}38 \%(5) \\
40 \text { months } \\
\text { (entry) }\end{array}$ & time & $\begin{array}{l}\text { Gender, male } \\
\text { Education } \uparrow \\
\text { Living situation, alone } \\
\text { Children living in the area, } \\
\text { number } \uparrow \\
\text { Cognitive impairment } \downarrow \\
\text { (MMSE) } \\
\text { BPSD (HRS), } \\
\text { presence of aggression, } \\
\text { hallucination, } \\
\text { depressive symptoms }\end{array}$ & $\begin{array}{l}1.64 \\
0.92 \\
1.92 \\
0.61 \\
0.93 \\
\\
4.17 \\
2.54 \\
1.07\end{array}$ & $\mathrm{HR}$ & & & & $\begin{array}{l}\text { AD-centre; } \\
\text { CPHR }\end{array}$ \\
\hline $\begin{array}{l}\text { Stevens et al. } \\
{[24] ; 2004 \text {; USA }}\end{array}$ & 215 & 74 & $6 / 7$ & $\begin{array}{l}\text { NINCDS- } \\
\text { ADRDA; } \\
\text { AD }\end{array}$ & $27 \%(6)$ & time & $\begin{array}{l}\text { Age } \uparrow \\
\text { Ethnicity, AA } \\
\text { BPSD } \uparrow(\mathrm{MBPC})\end{array}$ & $\begin{array}{l}1.04 \\
0.43 \\
1.06\end{array}$ & HR & $\begin{array}{l}\text { Family SES } \uparrow \\
\text { Distress, with } \\
\text { cognitive impair- } \\
\text { ment and BPSD, } \\
\text { with functional } \\
\text { status }\end{array}$ & $\begin{array}{l}1.02 \\
1.54 \\
1.56\end{array}$ & HR & $\begin{array}{l}\text { Memory } \\
\text { clinic; } \\
\text { CPHR }\end{array}$ \\
\hline $\begin{array}{l}\text { Banerjee et al. } \\
{[30] ; 2003 ;} \\
\text { UK }\end{array}$ & 100 & $\mathrm{n} / \mathrm{a}$ & $1 / 3$ & $\begin{array}{l}\mathrm{n} / \mathrm{a} \text {; } \\
\text { dementia }\end{array}$ & $\begin{array}{l}22 \%(0.5) \\
29 \%(1)\end{array}$ & risk & $\begin{array}{l}\text { Living situation, } \\
\text { with CG } \\
\text { BPSD } \uparrow\end{array}$ & $\begin{array}{l}0.05 \\
1.08\end{array}$ & OR & $\begin{array}{l}\text { Psychological } \\
\text { QoL } \downarrow \\
\text { (WHOQOL-BREF) }\end{array}$ & 1.10 & OR & $\begin{array}{l}\text { Psychiatric } \\
\text { services; } \\
\text { LR }\end{array}$ \\
\hline $\begin{array}{l}\text { Gaugler et al. } \\
{[25] ; 2003 ; \text { USA }} \\
\text { (MADDE) }\end{array}$ & 3,944 & $\mathrm{n} / \mathrm{a}$ & $3 / 7$ & $\begin{array}{l}\text { p.d.; } \\
\text { dementia }\end{array}$ & $\begin{array}{l}21 \%(1) \\
35 \%(2) \\
43 \%(3)\end{array}$ & time & $\begin{array}{l}\text { Age, } 90+ \\
\text { Gender, male } \\
\text { Ethnicity, } \\
\text { AA } \\
\text { Hispanic } \\
\text { Living situation, alone } \\
\text { Cognitive impairment } \uparrow \\
\text { (MMSE score }<11 \text { ) } \\
\text { Functional impairment } \uparrow \\
\text { (IADL) } \\
\text { BPSD } \uparrow\end{array}$ & $\begin{array}{l}1.46 \\
1.23 \\
0.52 \\
0.46 \\
1.55 \\
1.84 \\
1.94 \\
1.62\end{array}$ & HR & $\begin{array}{l}\text { Age, } 80+ \\
\text { Income, } \\
<\text { USD } 10,000 \\
\text { Burden } \uparrow(\mathrm{ZBI}>18) \\
\text { Health } \downarrow \\
\text { Unmet ADL/IADL } \\
\text { needs, }>2.5 \\
\text { Use of in-home } \\
\text { services } \downarrow \\
\text { ADS use } \\
\text { (ref. middle) } \downarrow, \uparrow\end{array}$ & $\begin{array}{l}1.80 \\
0.57 \\
1.54 \\
1.44 \\
1.18 \\
1.34 \\
1.30\end{array}$ & HR & $\begin{array}{l}\text { CLTC user; } \\
\text { CPHR }\end{array}$ \\
\hline $\begin{array}{l}\text { Young } \\
\text { [41]; 2003; USA }\end{array}$ & 532 & 77 & $2 / 4$ & $\begin{array}{l}\mathrm{n} / \mathrm{a} \\
\mathrm{AD}\end{array}$ & $28 \%(2)$ & risk & $\begin{array}{l}\text { Men: } \\
\text { Memory impairment } \downarrow \\
\text { Functional impair- } \\
\text { ment } \uparrow \text { (ADL) } \\
\text { BPSD } \uparrow \\
\text { Women: } \\
\text { Memory impairment } \downarrow \\
\text { Functional impairment } \downarrow \\
\text { (ADL) }\end{array}$ & $\begin{array}{l}0.81 \\
1.13 \\
1.03 \\
0.81 \\
1.11\end{array}$ & OR & $\begin{array}{l}\text { Men: } \\
\text { Intended placement } \\
\text { Social support } \uparrow \\
\text { Hours of care } \uparrow \\
\text { Women: } \\
\text { Intended placement }\end{array}$ & $\begin{array}{l}1.13 \\
0.99 \\
0.99 \\
1.19\end{array}$ & OR & $\begin{array}{l}\text { Support } \\
\text { group } \\
\text { meetings; } \\
\text { LR }\end{array}$ \\
\hline
\end{tabular}


Table 1 (continued)

\begin{tabular}{|c|c|c|c|c|c|c|c|c|c|c|c|c|c|}
\hline $\begin{array}{l}\text { Study; } \\
\text { year; } \\
\text { country }\end{array}$ & $\begin{array}{l}\mathrm{n}^{1} \mid \mathrm{n}^{2} \\
\mathrm{n}^{3}\end{array}$ & $\mathrm{~A}^{4}$ & $\begin{array}{l}\mathrm{OI} / \\
\mathrm{TP}^{5}\end{array}$ & $\begin{array}{l}\text { DS; } \\
D^{6}\end{array}$ & $\mathrm{IR}^{7}$ & $\begin{array}{l}\text { Time } \\
\text { and/or } \\
\text { risk }^{8}\end{array}$ & $\begin{array}{l}\text { CR factors }{ }^{9} \\
\left(\mathrm{I}^{10}\right)\end{array}$ & Risk $^{11}$ & $\begin{array}{l}{ }^{1} \text { Risk } \\
\text { type }\end{array}$ & $\begin{array}{l}\text { CG factors }{ }^{9} \\
\left(\mathrm{I}^{10}\right)\end{array}$ & Risk $^{11}$ & $\begin{array}{l}\text { Risk } \\
\text { type }\end{array}$ & $\begin{array}{l}\text { Methodical } \\
\text { notes }^{12}\end{array}$ \\
\hline $\begin{array}{l}\text { Yaffe et al. [26]; } \\
\text { 2002; USA } \\
\text { (MADDE) }\end{array}$ & 5,788 & 79 & $3 / 6$ & $\begin{array}{l}\text { p.d.; } \\
\text { dementia }\end{array}$ & $\begin{array}{l}22 \%(1) \\
40 \%(2) \\
52 \%(3)\end{array}$ & time & $\begin{array}{l}\text { Age, } 80+ \\
\text { Ethnicity, } \\
\text { AA } \\
\text { Hispanic } \\
\text { Living situation, alone } \\
\text { Cognitive impairment } \uparrow \\
\text { (MMSE score }<15 \text { ) } \\
\text { Functional impairment } \uparrow \\
\text { (ADL) } \\
\text { BPSD, presence }\end{array}$ & $\begin{array}{l}1.30 \\
0.60 \\
0.40 \\
1.74 \\
1.52 \\
1.38 \\
1.30\end{array}$ & HR & $\begin{array}{l}\text { Age, } 65+ \\
\text { Marital status, } \\
\text { married } \\
\text { Hours of care, } \\
\geq 90 \mathrm{~h} / \text { week } \\
\text { Functional } \\
\text { impairment } \uparrow \\
(\mathrm{ADL} / \mathrm{IADL}) \\
\text { Depression, } \geq 6 \\
\text { symptoms }(\mathrm{GDS}) \\
\text { Burden } \uparrow(\mathrm{ZBI} \geq 20)\end{array}$ & $\begin{array}{l}1.17 \\
1.16 \\
1.12 \\
\\
1.16 \\
1.18 \\
1.79\end{array}$ & HR & $\begin{array}{l}\text { CLTC user; } \\
\text { CPHR }\end{array}$ \\
\hline $\begin{array}{l}\text { Andrieu et al. } \\
{[61] ; 2001 ;} \\
\text { France (ELSA) }\end{array}$ & 318 & $\begin{array}{l}45-89 \\
75\end{array}$ & $1 / 3$ & $\begin{array}{l}\text { NINCDS- } \\
\text { ADRDA; } \\
\text { AD }\end{array}$ & $20 \%(1)$ & risk & $\begin{array}{l}\text { Nutritional status } \downarrow \text { (MNA) } \\
\text { Eating behavioural } \\
\text { problems, overeating }\end{array}$ & $\begin{array}{l}2.31 \\
5.56\end{array}$ & OR & & & & $\begin{array}{l}\text { Memory } \\
\text { clinic; } \\
\text { LR }\end{array}$ \\
\hline $\begin{array}{l}\text { Hébert et al. } \\
\text { [15]; 2001; } \\
\text { Canada } \\
\text { (CSHA) }\end{array}$ & 326 & $65+$ & $5 / 3$ & $\begin{array}{l}\text { NINCDS- } \\
\text { ADRDA, } \\
\text { ICD-10, } \\
\text { DSM-III-R; } \\
\text { AD, VD, } \\
\text { OD }\end{array}$ & $\begin{array}{l}51 \%(5) \\
41 \text { months } \\
\text { (entry) }\end{array}$ & time & $\begin{array}{l}\text { Type of dementia, AD } \\
\text { Functional impairment } \uparrow \\
\text { (ADL) } \\
\text { BPSD } \uparrow(\mathrm{DBD}) \\
\text { CG relationship, } \\
\text { not spouse or child }\end{array}$ & $\begin{array}{l}1.83 \\
4.02 \\
1.02 \\
1.55\end{array}$ & HR & $\begin{array}{l}\text { Age, } 60+ \\
\text { Burden } \uparrow(\mathrm{ZBI}>18) \\
\text { Health problems, } \\
>4 \\
\text { Desire to } \\
\text { institutionalise }\end{array}$ & $\begin{array}{l}1.83 \\
1.71 \\
2.60 \\
4.74\end{array}$ & HR & $\begin{array}{l}\text { Population- } \\
\text { based } \\
\text { sample; } \\
\text { CPHR }\end{array}$ \\
\hline $\begin{array}{l}\text { Pot et al. } \\
{[16] ; 2001 ;} \\
\text { The } \\
\text { Netherlands } \\
\text { (AMSTEL) }\end{array}$ & 138 & 78 & $1 / 2$ & $\begin{array}{l}\text { CAMDEX; } \\
\text { dementia }\end{array}$ & $33 \%(1)$ & risk & $\begin{array}{l}\text { CG relationship, } \\
\text { non-spouse } \\
\text { Functional impairment } \uparrow\end{array}$ & $\begin{array}{l}\uparrow \\
\uparrow\end{array}$ & & $\begin{array}{l}\text { Education level } \uparrow \\
\text { Pressure from care } \uparrow \\
\text { (SPPIC) } \\
\text { Extraverted non- } \\
\text { spouse (EPQ-R) }\end{array}$ & $\begin{array}{l}\uparrow \\
\uparrow \\
\uparrow\end{array}$ & & $\begin{array}{l}\text { Population- } \\
\text { based } \\
\text { sample; } \\
\text { LR }\end{array}$ \\
\hline $\begin{array}{l}\text { Smith et al. } \\
{[18] ; 2001 ; \text { USA }} \\
\text { (ADPR) }\end{array}$ & 512 & $\begin{array}{l}65-98 \\
80\end{array}$ & $\begin{array}{l}5 / \\
\mathrm{n} / \mathrm{a}\end{array}$ & $\begin{array}{l}\text { NINCDS- } \\
\text { ADRDA, } \\
\text { DSM-III-R; } \\
\text { AD, VD, } \\
\text { MD }\end{array}$ & $\begin{array}{l}39 \%(5) \\
81 \text { months } \\
\text { (onset) }\end{array}$ & $\begin{array}{l}\text { time/ } \\
\text { risk }\end{array}$ & $\begin{array}{l}\text { Age } \uparrow \\
\text { Gender, male } \\
\text { Marital status, } \\
\text { single } \\
\text { widowed } \\
\text { Living situation, } \\
\text { with spouse } \\
\text { Severity of dementia } \uparrow \\
\text { (DRS) } \\
\text { Functional impairment } \uparrow \\
\text { (ADL/IADL) }\end{array}$ & $\begin{array}{l}1.03 \\
0.50 \\
2.50 \\
1.60 \\
0.40 \\
\uparrow \\
\uparrow\end{array}$ & $\mathrm{HR}$ & & & & $\begin{array}{l}\text { AD patient } \\
\text { registry; } \\
\text { CPHR }\end{array}$ \\
\hline $\begin{array}{l}\text { Spruytte et al. } \\
\text { [34]; 2001; } \\
\text { Belgium }\end{array}$ & 144 & $\begin{array}{l}61-94 \\
82\end{array}$ & $1 / 2$ & $\begin{array}{l}\text { p.d.; } \\
\text { dementia }\end{array}$ & $15 \%(0.75)$ & risk & $\begin{array}{l}\text { Functional impairment } \downarrow \\
\text { (MAS-D) } \\
\text { Quality of CG relation- } \\
\text { ship, more warmth, less } \\
\text { conflict and critique }\end{array}$ & 1.09 & OR & $\begin{array}{l}\text { Desire to institution- } \\
\text { alise (DIS) } \\
\text { Structural changes in } \\
\text { material CG } \\
\text { situation } \uparrow\end{array}$ & 1.53 & OR & $\begin{array}{l}\text { CLTC user; } \\
\text { LR }\end{array}$ \\
\hline $\begin{array}{l}\text { Smith et al. } \\
\text { [17]; 2000; USA } \\
\text { (REP) }\end{array}$ & 220 & $\begin{array}{l}65+ \\
81\end{array}$ & $\begin{array}{l}8 / \\
n / a\end{array}$ & $\begin{array}{l}\text { NINCDS- } \\
\text { ADRDA, } \\
\text { DSM-III-R; } \\
\text { AD, VD, } \\
\text { MD }\end{array}$ & $\begin{array}{l}90 \%(8) \\
30 \text { months } \\
\text { (onset) }\end{array}$ & time & $\begin{array}{l}\text { Age } \uparrow \\
\text { Education, } \\
\text { number of years } \uparrow \\
\text { Marital status, single } \\
\text { Living situation, } \\
\text { retirement } \\
\text { assisted living } \\
\text { Change in comorbidity } \uparrow \\
\text { (C-Score) } \\
\text { Change in daily assistance } \uparrow\end{array}$ & $\begin{array}{l}1.70 \\
0.96 \\
1.90 \\
\\
2.20 \\
2.20 \\
1.90 \\
2.20\end{array}$ & $\mathrm{HR}$ & & & & $\begin{array}{l}\text { Data files of } \\
\text { outpatients; } \\
\text { CPHR }\end{array}$ \\
\hline $\begin{array}{l}\text { Fisher and } \\
\text { Lieberman [45]; } \\
\text { 1999; USA }\end{array}$ & 164 & 77 & $2 / 5$ & $\begin{array}{l}\text { NINCDS- } \\
\text { ADRDA; } \\
\text { AD }\end{array}$ & $48 \%(2)$ & risk & & & & $\begin{array}{l}\text { Family feelings, } \\
\text { negative } \\
\text { Emotional } \\
\text { closeness } \uparrow \\
\text { Family efficiency } \uparrow\end{array}$ & $\begin{array}{l}1.47 \\
1.64 \\
0.67 \\
\end{array}$ & OR & $\begin{array}{l}\text { Memory } \\
\text { clinic; } \\
\text { LR }\end{array}$ \\
\hline $\begin{array}{l}\text { Miller et al. } \\
\text { [29]; 1999; USA } \\
\text { (CERAD } \\
\text { project) }\end{array}$ & $\begin{array}{l}122 \\
(\mathrm{AA})\end{array}$ & 74 & $7 / 5$ & $\begin{array}{l}\text { NINCDS- } \\
\text { ADRDA; } \\
\text { AD }\end{array}$ & $25 \%(3.4)$ & time & $\begin{array}{l}\text { Marital status, unmarried } \\
\text { Severity of dementia } \uparrow \\
\text { (CDR) } \\
\text { Functional impairment } \uparrow \\
\text { (ADL) }\end{array}$ & $\begin{array}{l}3.23 \\
1.64 \\
1.27\end{array}$ & $\mathrm{HR}$ & & & & $\begin{array}{l}\text { University } \\
\text { program; } \\
\text { CPHR }\end{array}$ \\
\hline
\end{tabular}


Table 1 (continued)

\begin{tabular}{|c|c|c|c|c|c|c|c|c|c|c|c|c|c|c|}
\hline $\begin{array}{l}\text { Study; } \\
\text { year; } \\
\text { country }\end{array}$ & $\begin{array}{r}\mathrm{n}^{1} \\
\mathrm{n}\end{array}$ & $\begin{array}{l}\mathrm{n}^{2} \\
\mathrm{n}^{3}\end{array}$ & $\mathrm{~A}^{4}$ & $\begin{array}{l}\mathrm{OI} / \\
\mathrm{TP}^{5}\end{array}$ & $\begin{array}{l}\text { DS; } \\
\mathrm{D}^{6}\end{array}$ & $\mathrm{IR}^{7}$ & $\begin{array}{l}\text { Time } \\
\text { and/or } \\
\text { risk }^{8}\end{array}$ & $\begin{array}{l}\text { CR factors }{ }^{9} \\
\left(\mathrm{I}^{10}\right)\end{array}$ & $\operatorname{Risk}^{11}$ & $\begin{array}{l}{ }^{1} \text { Risk } \\
\text { type }\end{array}$ & $\begin{array}{l}\text { CG factors }{ }^{9} \\
\left(\mathrm{I}^{10}\right)\end{array}$ & Risk $^{11}$ & $\begin{array}{l}\text { Risk } \\
\text { type }\end{array}$ & $\begin{array}{l}\text { Methodical } \\
\text { notes }^{12}\end{array}$ \\
\hline $\begin{array}{l}\text { Bannister et al. } \\
\text { [53]; 1998; } \\
\text { UK }\end{array}$ & & 116 & $\begin{array}{l}65+ \\
80\end{array}$ & $1 / 12$ & $\begin{array}{l}\text { NINCDS- } \\
\text { ADRDA, } \\
\text { DSM-III-R; } \\
\text { AD, VD, } \\
\text { LTD }\end{array}$ & $22 \%(1)$ & risk & $\begin{array}{l}\text { Cognitive impairment } \uparrow \\
\text { (CAMCOG) }\end{array}$ & $\uparrow$ & & $\begin{array}{l}\text { Active coping } \\
\text { strategies } \downarrow \text { (CSS) }\end{array}$ & $\uparrow$ & & $\begin{array}{l}\text { Memory } \\
\text { clinic/ } \\
\text { psychiatry } \\
\text { service; } \\
\text { LR }\end{array}$ \\
\hline $\begin{array}{l}\text { Hope et al. [62]; } \\
\text { 1998; UK }\end{array}$ & & 100 & $65+$ & $1 / 3$ & $\begin{array}{l}\text { NINCDS- } \\
\text { ADRDA, } \\
\text { DSM-III-R; } \\
\text { AD, VD }\end{array}$ & $\mathrm{n} / \mathrm{a}$ & risk & $\begin{array}{l}\text { BPSD, nighttime activity } \uparrow \\
\text { (PBE) } \\
\text { Physical status, immobility, } \\
\text { difficulty in walking } \uparrow \\
\text { (PBE) } \\
\text { Away from CG, } \\
>16 \text { h/week }\end{array}$ & $\begin{array}{l}\uparrow \\
\uparrow \\
\uparrow \\
\uparrow\end{array}$ & & Gender, female & $\uparrow$ & & $\begin{array}{l}\text { Geriatric } \\
\text { health care; } \\
\text { classifica- } \\
\text { tion tree }\end{array}$ \\
\hline $\begin{array}{l}\text { Miller et al. } \\
\text { [37]; 1998; USA } \\
\text { (CERAD } \\
\text { project) }\end{array}$ & 639 & & 71 & $9 / 18$ & $\begin{array}{l}\text { NINCDS- } \\
\text { ADRDA; } \\
\text { AD }\end{array}$ & $\begin{array}{l}59 \%(9) \\
41 \text { months } \\
\text { (entry) }\end{array}$ & time & $\begin{array}{l}\text { Severity of dementia } \uparrow \\
\text { (CDR) } \\
\text { For the first } 3 \text { years of the } \\
\text { study: } \\
\text { Married persons: } \\
\text { Age } \uparrow \\
\text { Cognitive impairment } \downarrow \\
\text { (MMSE) } \\
\text { Functional impairment } \uparrow \\
\text { (ADL/IADL) } \\
\text { HHA/100,000 persons } \\
\text { (65+), number } \uparrow \\
\text { NH beds/1,000 persons } \\
\text { (85+), number } \uparrow \\
\text { NH occupancy rates } \uparrow \\
\text { Unmarried persons: } \\
\text { Medicaid LTC use } \uparrow\end{array}$ & $\begin{array}{l}2.44 \\
\\
1.04 \\
0.96 \\
1.15 \\
0.77 \\
1.01 \\
1.18 \\
0.90\end{array}$ & HR & & & & $\begin{array}{l}\text { University } \\
\text { program; } \\
\text { CPHR }\end{array}$ \\
\hline $\begin{array}{l}\text { Wells and Over } \\
\text { [46]; 1998; } \\
\text { Australia }\end{array}$ & & $\begin{array}{l}93 \\
\text { spou- } \\
\text { ses }\end{array}$ & $\begin{array}{l}60+ \\
75\end{array}$ & $1.5 / 2$ & $\begin{array}{l}\text { p.d.; } \\
\text { dementia }\end{array}$ & $42 \%(1.5)$ & time & & & & $\begin{array}{l}\text { Coping strategies: } \\
\text { Confrontative } \\
\text { coping } \uparrow \\
\text { Seeks social } \\
\text { support } \uparrow \\
\text { Accepts } \\
\text { responsibility } \uparrow\end{array}$ & $\begin{array}{l}2.12 \\
1.91 \\
0.28\end{array}$ & OR & $\begin{array}{l}\text { ADS, geri- } \\
\text { atric health } \\
\text { care; } \\
\text { LR }\end{array}$ \\
\hline $\begin{array}{l}\text { Heyman et al. } \\
\text { [27]; 1997; USA } \\
\text { (CERAD } \\
\text { project) }\end{array}$ & 727 & & 72 & $6 / 6$ & $\begin{array}{l}\text { NINCDS- } \\
\text { ADRDA; } \\
\text { AD }\end{array}$ & $\begin{array}{l}\mathrm{n} / \mathrm{a} \\
42 \text { months } \\
\text { (entry) }\end{array}$ & time & $\begin{array}{l}\text { Age } \uparrow \\
\text { Gender/marital status, } \\
\text { unmarried men } \\
\text { Severity of dementia } \uparrow \\
\text { (CDR) } \\
\text { Cognitive impairment } \uparrow \\
\text { (MMSE) } \\
\text { Functional impairment } \uparrow \\
\text { (ADL) }\end{array}$ & $\begin{array}{l}1.17 \\
2.11 \\
1.24 \\
1.18 \\
1.30\end{array}$ & HR & & & & $\begin{array}{l}\text { University } \\
\text { program; } \\
\text { CPHR }\end{array}$ \\
\hline $\begin{array}{l}\text { Scott et al. [33]; } \\
\text { 1997; USA }\end{array}$ & 786 & & 82 & $1.5 / 2$ & $\begin{array}{l}\mathrm{n} / \mathrm{a} ; \\
\mathrm{AD}, \mathrm{MD}\end{array}$ & $8 \%(1.5)$ & risk & $\begin{array}{l}\text { Ethnicity (ref. AA), } \\
\text { white American } \\
\text { CG relationship (ref. } \\
\text { spouse), child } \\
\text { relative } \\
\text { Type of dementia (ref. AD), } \\
\text { VD } \\
\text { OD } \\
\text { Change in functional status } \\
\downarrow(A D L)\end{array}$ & $\begin{array}{l}3.30 \\
4.80 \\
1.20 \\
\\
0.40 \\
0.50 \\
3.50\end{array}$ & OR & & & & $\begin{array}{l}\text { CLTC user; } \\
\text { LR }\end{array}$ \\
\hline $\begin{array}{l}\text { Severson et al. } \\
\text { [19]; 1994; USA } \\
\text { (ADPR) }\end{array}$ & 133 & & $\begin{array}{l}60+ \\
80\end{array}$ & $4 / 3$ & $\begin{array}{l}\text { NINCDS- } \\
\text { ADRDA, } \\
\text { DSM-III-R; } \\
\text { AD, VD, } \\
\text { MD, OD }\end{array}$ & $\begin{array}{l}40 \%(4) \\
67 \text { months } \\
\text { (onset) }\end{array}$ & risk & $\begin{array}{l}\text { Marital status, unmarried } \\
\text { Cognitive impairment } \uparrow \\
\text { (DRS) } \\
\text { Functional impairment } \uparrow \\
\text { (ADL) }\end{array}$ & $\begin{array}{l}2.70 \\
2.10 \\
1.70\end{array}$ & $\mathrm{HR}$ & & & & $\begin{array}{l}\text { AD patient } \\
\text { registry; } \\
\text { CPHR }\end{array}$ \\
\hline
\end{tabular}


Table 1 (continued)

\begin{tabular}{|c|c|c|c|c|c|c|c|c|c|c|c|c|}
\hline $\begin{array}{l}\text { Study; } \\
\text { year; } \\
\text { country }\end{array}$ & $\begin{array}{l}\mathrm{n}^{1} \mid \mathrm{n}^{2} \\
\mathrm{n}^{3}\end{array}$ & $\mathrm{~A}^{4}$ & $\begin{array}{l}\mathrm{OI} / \\
\mathrm{TP}^{5}\end{array}$ & $\begin{array}{l}\text { DS; } \\
D^{6}\end{array}$ & $\mathrm{IR}^{7}$ & $\begin{array}{l}\text { Time } \\
\text { and/or } \\
\text { risk }^{8}\end{array}$ & $\begin{array}{l}\text { CR factors }{ }^{9} \\
\left(I^{10}\right)\end{array}$ & $\begin{array}{r}\text { Risk }^{11} \text { Risk } \\
\text { type }\end{array}$ & $\begin{array}{l}\text { CG factors }{ }^{9} \\
\left(\mathrm{I}^{10}\right)\end{array}$ & Risk $^{11}$ & $\begin{array}{l}\text { Risk } \\
\text { type }\end{array}$ & $\begin{array}{l}\text { Methodical } \\
\text { notes }^{12}\end{array}$ \\
\hline $\begin{array}{l}\text { Wright [63]; } \\
\text { 1994; USA }\end{array}$ & $\begin{array}{c}30 \\
\text { couples }\end{array}$ & 68 & $2 / 2$ & $\begin{array}{l}\text { p.d.; } \\
\text { AD }\end{array}$ & $28 \%(2)$ & risk & $\begin{array}{l}\text { Educational level } \uparrow \\
\text { Marital relationship, } \\
\text { unhappy (DMARS) }\end{array}$ & $\begin{array}{l}\uparrow \\
\uparrow\end{array}$ & $\begin{array}{l}\text { Commitment to } \\
\text { future } \uparrow \\
\text { Physical health } \uparrow \\
\text { (MAI) } \\
\text { CG time } \downarrow\end{array}$ & $\begin{array}{l}\downarrow \\
\downarrow \\
\downarrow\end{array}$ & & $\begin{array}{l}\text { AD-centre, } \\
\text { GP; } \\
\text { discrimi- } \\
\text { nant } \\
\text { analysis }\end{array}$ \\
\hline $\begin{array}{l}\text { Haupt and } \\
\text { Kurz } \\
\text { [40]; 1993; } \\
\text { Germany }\end{array}$ & 66 & $\begin{array}{l}56-89 \\
73\end{array}$ & $1 / 2$ & $\begin{array}{l}\text { ICD-10; } \\
\text { AD }\end{array}$ & $33 \%(1)$ & risk & $\begin{array}{l}\text { Age } \uparrow \\
\text { Cognitive impair- } \\
\text { ment } \uparrow \text { (CAMCOG) } \\
\text { BPSD, incontinence, } \\
\text { aggression, angry outbursts, } \\
\text { depression, presence } \\
\text { (CAMDEX) }\end{array}$ & $\begin{array}{l}\uparrow \\
\uparrow \\
\uparrow \\
\uparrow\end{array}$ & $\begin{array}{l}\text { Wish to leave the } \\
\text { care to someone else }\end{array}$ & $\uparrow$ & & $\begin{array}{l}\text { Outpatients; } \\
\text { discrimi- } \\
\text { nant } \\
\text { analysis }\end{array}$ \\
\hline $\begin{array}{l}\text { O’Donnell } \\
\text { et al. [20]; 1992; } \\
\text { USA }\end{array}$ & 143 & 71 & $1.5 / 2$ & $\begin{array}{l}\text { NINCDS- } \\
\text { ADRDA, } \\
\text { CT scan; } \\
\text { AD, VD, MD }\end{array}$ & $\begin{array}{l}36 \%(1.5) \\
88 \text { months } \\
\text { (onset) }\end{array}$ & risk & $\begin{array}{l}\text { Severity of dementia } \uparrow \\
\text { BPSD, paranoia, } \\
\text { incontinence, aggression, } \\
\text { delusions, hallucinations, } \\
\text { presence }\end{array}$ & $\uparrow$ & & & & $\begin{array}{l}\text { AD-centre; } \\
\text { CPHR }\end{array}$ \\
\hline $\begin{array}{l}\text { Lieberman and } \\
\text { Kramer [64]; } \\
\text { 1991; USA }\end{array}$ & 321 & 74 & $1 / 2$ & $\begin{array}{l}\text { p.d.; } \\
\text { AD, VD, OD }\end{array}$ & $22 \%(1)$ & risk & CG relationship, spouse & $\downarrow$ & $\begin{array}{l}\text { Distress, caused by } \\
\text { family problems } \uparrow\end{array}$ & $\uparrow$ & & $\begin{array}{l}\text { AD-centre; } \\
\text { LR }\end{array}$ \\
\hline $\begin{array}{l}\text { Pruchno et al. } \\
\text { [12]; 1990; USA }\end{array}$ & 220 & $\mathrm{n} / \mathrm{a}$ & $1 / 2$ & $\begin{array}{l}\mathrm{n} / \mathrm{a} \\
\mathrm{AD}, \mathrm{OD}\end{array}$ & $22 \%(1)$ & risk & Cognitive impairment $\uparrow$ & $2.32 \mathrm{OR}$ & $\begin{array}{l}\text { Religion, Jewish } \\
\text { Desire to } \\
\text { institutionalise } \\
\text { Duration as CG } \downarrow\end{array}$ & $\begin{array}{l}1.16 \\
1.79 \\
2.80\end{array}$ & OR & $\begin{array}{l}\text { Gate- } \\
\text { keepers; } \\
\text { LR }\end{array}$ \\
\hline $\begin{array}{l}\text { Knopman et al. } \\
\text { [65]; 1988; USA }\end{array}$ & 101 & $\begin{array}{l}56-87 \\
71\end{array}$ & $2 / 2$ & $\begin{array}{l}\text { NINCDS- } \\
\text { ADRDA; } \\
\text { AD }\end{array}$ & $\begin{array}{l}\text { mild: } \\
12 \%(1) \\
35 \%(2) \\
\text { advanced: } \\
39 \%(1) \\
62 \%(2)\end{array}$ & risk & $\begin{array}{l}\text { BPSD, nighttime awaking, } \\
\text { irritability, presence } \\
\text { Functional impairment } \uparrow \\
\text { (ADL) }\end{array}$ & $\uparrow$ & & & & $\begin{array}{l}\text { AD-centre; } \\
\text { LR }\end{array}$ \\
\hline $\begin{array}{l}\text { Colerick and } \\
\text { George [66]; } \\
\text { 1986; USA }\end{array}$ & 209 & & $1 / 2$ & $\begin{array}{l}\mathrm{n} / \mathrm{a} \\
\mathrm{AD}\end{array}$ & $22 \%(1)$ & risk & CG relationship, spouse & $\downarrow$ & & & & $\begin{array}{l}\text { University } \\
\text { program; } \\
\text { LR }\end{array}$ \\
\hline
\end{tabular}

$\mathrm{AA}=$ African Americans; $\mathrm{AD}$-centre = $\mathrm{AD}$ diagnostic centre; $\mathrm{ADL}=$ activities of daily living; $\mathrm{ADPR}=\mathrm{AD}$ Patient Registry; ADS = adult day services; AMSTEL = Amsterdam Study of the Elderly; BRS-D = Behaviour Rating ScaleDementia; CAMCOG = Cambridge Cognitive Examination; CAMDEX = Cambridge Mental Disorders of the Elderly Exam; CDR $=$ Clinical Dementia Rating; CERAD = Consortium to Establish a Registry for AD; CES-D = Centre for Epidemiological Studies Depression Index; CG = caregiver; CPHR = Cox proportional hazards regression; $\mathrm{CR}=$ care recipient; $\mathrm{C}$-Score $=$ Charlston score; CSHA = Canadian Study of Health and Aging; CSS = Carers Stress Scale; $\mathrm{DBD}=$ Dementia Behaviour Disturbance Scale; DEPAD = Outcome of Community-Dwelling Dementia Patients; DIS = Desire to Institutionalise Scale; DMARS = Dyadic Marital Adjustment Rating Scale; DRS = Dementia Rating Scale; DSM = Diagnostic and Statistical Manual of Mental Disorders; ELSA = Etude Longitudinal de Suivi d'Alzheimer; EPQ-R = Extraversion Personality Questionnaire, revised version; FTD = frontotemporal dementia; GDS = Geriatric Depression Scale; HHA = Home Health Agencies; HR = hazard ratio; HRS $=$ Hamilton Rating Scale; IADL = instrumental activities of daily living; ICD = International Statistical Classification of Diseases; LR = logistic regression; LTC = long-term care; LTD = Lewy-type dementia; MAASBED = Maastricht Study of Behaviour in Dementia; MADDE = Medicare Alzheimer's Disease Demonstration Evaluation; MAI = Multilevel Assessment Inventory; MAS-D = Mechelse Activiteiten Schaal-versie Dementie; $\mathrm{MBPC}=$ Memory and Behaviour Problem Checklist; $\mathrm{MD}=$ mixed dementia; $\mathrm{MDS}-\mathrm{HC}=$ Minimum
Data Set-Home Care; MMSE = Mini-Mental State Examination; MNA = MiniNutritional Assessment; $\mathrm{n} / \mathrm{a}=$ not available; $\mathrm{NH}=$ nursing home; NINCDSADRDA = National Institute of Neurological and Communicative DisordersAlzheimer's Disease and Related Disorders Association; NLCS = National Longitudinal Caregiver Study; NPI = Neuropsychiatric Inventory; OD = dementia not otherwise specified; $\mathrm{OR}=$ odds ratio; $\mathrm{PBE}=$ Present Behavioural Examination; p.d. = physician's diagnosis; $\mathrm{QoL}=$ quality of life; RBDS $=$ RosowBreslau Disability Scale; ref. = reference; REP $=$ Rochester Epidemiology Project; SES = socio-economic status; SF-36 = 36-item Short Form Health Survey; SPPIC = Self-Perceived Pressure from Informal Care; VD = vascular dementia; WHOQOL-BREF = World Health Organisation quality of lifeBREF; ZBI = Zarit Burden Inventory.

${ }^{1}$ Size of sample of dementia sufferers. ${ }^{2}$ Size of sample of caregivers. ${ }^{3}$ Size of sample of dyads of dementia sufferers and caregivers. ${ }^{4}$ Age range and/or mean age in years at baseline. ${ }^{5}$ Observation interval/number of observation timepoints. ${ }^{6}$ Diagnosticsystemand diagnoses. ${ }^{7}$ Cumulativeinstitutionalisation rate in percent (observation interval in years) and/or mean time in months to institutionalisation from entry in the study or from estimated onset of dementia. ${ }^{8}$ Focusing on time to and/or risk of institutionalisation. ${ }^{9}$ Factors of care recipients/caregivers influencing institutionalisation, $\uparrow$ for high level, $\downarrow$ for low level. ${ }^{10}$ Instruments measuring factors. ${ }^{11}$ If specified, value of risk, otherwise $\uparrow$ for increased risk of institutionalisation and $\downarrow$ for decreased risk of institutionalisation. ${ }^{12}$ Kind of sample and of multivariate data analysis. 
ies. One year following entry in the studies, the highest reported admission rates were between 20 and $25 \%$ for AD centre, MC and CLTC samples. Pot et al. [16] reported an admission rate of $33 \%$ after 1 year for a populationbased sample. After 2 years, admission rates were also between 33 and $50 \%$ for AD centre, MC and CLTC samples. For longer observation intervals, admission rates increased only slightly. Hébert et al. [15] and Luck et al. [14] found admission rates of 51 and $48 \%$ after 5 and 6 years for population-based samples. Smith et al. [17] reported an admission rate of $90 \%$ after 8 years, derived from health care records.

A few studies reported the mean time until institutionalisation. Basically, the mean time from study entry to NHP ranged from 30 to 40 months, obtained from data files and AD centre/MC samples. For a population-based sample, the mean time from the baseline interview to NHP was 41 months [15]. A few studies additionally reported the mean time from the estimated onset of the disease to NHP ranging from 30 to 88 months, obtained from data files and samples of AD centres [17-20]. Luck et al. [14] reported a mean time for incident dementia cases from diagnosis of dementia to NHP of 35 months for a population-based sample.

\section{Factors Influencing Institutionalisation of Persons with Dementia}

As predisposing variables affecting institutionalisation of persons with dementia, sociodemographic and relationship characteristics of patients and caregivers were analysed. A great number of studies found an increased risk of and/or a shorter time to NHP for advanced age of patients (hazard ratio of risk: 1.48-2.00 [21, 22]; hazard ratio of time: 1.01-1.70 [17, 23-27]) and caregivers (hazard ratio of time: $1.01-1.83[15,23,25,26])$, for male (hazard ratio of time: 1.23-1.64 [23, 25, 28]), for unmarried (single, widowed or divorced) patients (hazard ratio of time: $1.60-4.50$, reference: married $[14,17,18,29])$ and for patients living alone (hazard ratio of time: 1.55-1.92 $[25,26,28])$ compared to living with the spouse (hazard ratio of time: 0.40 [18]) or with the caregiver (odds ratio of risk: $0.05-0.79$ [30, 31]). A further factor to be analysed was the kin- and relationship of care recipients and caregivers. Studies showed an earlier institutionalisation when the caregiver was a child (hazard ratio of time: 3.10 [32]; odds ratio of risk: 4.80 [33]) or another relative (hazard ratio of time: 1.55 [15]; odds ratio of risk: 1.20 [33]) compared to the spouse. A good relationship decreased the risk of NHP (odds ratio: 0.92 [34]). A few studies conducted in the USA focusing on ethnicity of patients found a decreased risk for and/or an extended time to NHP for African Americans (hazard ratio of time: 0.43-0.68 [2326]) and for Hispanics (hazard ratio of time: 0.40-0.46 $[25,26])$ compared to white Americans (odds ratio of risk: 1.92-3.30 [31, 33]). Regarding socio-economic status, some studies showed an extended time to NHP for patients with a higher level of education (hazard ratio: 0.92$0.96[17,28])$. Caregivers who are employed [35] have a higher level of education [16] or a higher income (hazard ratio of risk: 1.15 [36]; odds ratio of risk: 1.02 [31]) institutionalised their care recipients sooner.

According to our conceptual framework, need variables comprise primary or secondary stressors leading to actual institutionalisation by the caregivers. The dementia-related cognitive, functional and behavioural changes as well as objective characteristics of the caregiving situation were considered as primary stressors. Institutionalisation of persons with dementia is associated with greater severity of dementia (hazard ratio of risk: 1.80 [22]; hazard ratio of time: $1.24-2.44[27,29,37])$, greater cognitive (hazard ratio of risk: 1.80-4.59 [19, 22, 38]; hazard ratio of time: 1.18-1.84 [25-27]) and functional impairment (hazard ratio of risk: 1.30-1.70 [19, 22]; hazard ratio of time: $1.27-4.02[15,23,25-27,29])$ and other behavioural and psychological dementia-related problems (hazard ratio of time: 1.02-1.62 [15, 24-26]) including symptoms such as aggression (hazard ratio of time: 4.17 [28]), depression (hazard ratio of risk: 1.60 [22]; hazard ratio of time: 1.07 [28]), hallucinations (hazard ratio of risk: 1.06 [39]; hazard ratio of time: 2.54 [28]) and incontinence $[20,40]$. If caregivers report many unmet needs of their care recipient in activities of daily living, they tend to institutionalise them sooner (odds ratio of risk: 1.77 [31]; hazard ratio of time: 1.18 [25]). Regarding type of dementia, the results showed an increased risk of and a shorter time to NHP for AD patients (odds ratio of risk for vascular dementia: 0.40 [33], and for dementia not otherwise specified: 0.50 [33]; hazard ratio of time: 1.83 [15]).

Another group of primary stressors were the objective characteristics of the caregiving situation. Regarding the association of weekly caregiving hours and institutionalisation, results were inconsistent. Whereas Gaugler et al. [36] and Yaffe et al. [26] reported earlier NHP with more caregiving hours (hazard ratio of risk: 1.01; hazard ratio of time: 1.12), Young [41] showed a higher risk for institutionalisation with fewer caregiving hours (odds ratio of risk: 0.99 ). The results were undisputed due to the caregivers' health. A higher rate of institutionalisation was associated with: increased health problems (hazard ratio of time: $1.44-2.60[15,25])$, presence of depression 
(hazard ratio of time and risk: $1.18[26,36])$ and dependencies in activities of daily living (odds ratio of risk: 1.11 [31]; hazard ratio of time: 1.16 [26]).

The secondary stressors included the subjective appraisals of caregiving stressors - especially caregivers' burden - life satisfaction and perceived quality of life. The burden of caregivers was often recorded by the Zarit Burden Inventory [42]. Some studies reported that a Zarit Burden Inventory score greater than 18 or 20 is associated with a higher risk of (hazard ratio: 2.47 [38]) or a shorter time (hazard ratio: $1.54-1.79[15,25,26])$ to institutionalisation. Moreover, the experience of greater distress with BPSD was also linked with an earlier NHP (hazard ratio: 1.54-2.70 [24, 32]). The risk of institutionalisation also increased with the perception of lower life satisfaction (hazard ratio: 1.52 [39]) and lower health-related quality of life (odds ratio: 1.10-6.40 [30, 43]).

Under enabling variables, we subsumed personal and social resources as well as community-based care services available to the caregivers; more precisely, formal and informal support used by the caregivers, as well as existing coping strategies of the caregivers for the management of difficult caregiving situations. Inconsistent results were found in predicting NHP by the use of adult day services and in-home help. Gaugler et al. [25] showed a curvilinear effect; rare and frequent use of adult day care was associated with earlier institutionalisation (hazard ratio of time: 1.30). Others showed earlier NHP for more frequent adult day service use (hazard ratio of risk: 1.01 [31]; hazard ratio of time: 1.33 [23]). In contrast, increased use of in-home help services was associated with lower risk of institutionalisation (hazard ratio: 0.83 [44]; for decreased use of in-home help services, hazard ratio of time: 1.34 [25]). Moreover, perception of social support and family efficiency by the caregivers decreased risk of NHP (odds ratio: 0.67-0.99 [41, 45]). Institutional placement was more likely when the caregiver employed coping strategies such as confrontation, reliance on social support or unwillingness to accept responsibility [46].

Finally, as expected, the desire to institutionalise led to increased risk of (odds ratio: 1.13-1.79 [12, 34, 41]) and shortened time (hazard ratio: 4.74 [15]) to actual institutionalisation.

\section{Discussion}

The aim of the study was to systematically review all studies analysing predictors of actual institutionalisation of persons with dementia. Forty-two studies worldwide fulfilled the inclusion criteria providing findings on rates of and time to institutionalisation of persons with dementia and analysing factors influencing institutionalisation.

The institutionalisation rate of persons with dementia increased from almost $20 \%$ in the first year after diagnosis of dementia to around $50 \%$ after 5 years, up to $90 \%$ after 8 years, and appreciably exceeded the institutionalisation rate in elderly population-based samples, which was reported to be $6-7 \%$ at 3 years [5]. The high institutionalisation rates in dementia were also supported by the finding that dementia is the strongest predictor for NHP after adjusting for comorbidity and functional impairment [5]. The risk of NHP increased fivefold for demented subjects compared to non-demented controls [47, 48]. It was assumed that the characteristics of dementia (e.g. cognitive impairment, higher dependencies, and behavioural problems) were responsible for the high risk of institutionalisation. Our findings regarding dementia-related symptoms supported this explanation.

A few studies examined the median time to institutionalisation. They reported similar results. Differences were caused by varying methodological approaches: most of the studies reported the median time from study entry to institutionalisation without information about the onset of the disease. However, the calculated median time depends on the point in the course of the disease when the individual was affiliated with the study. To deal with the problem, a few studies additionally reported the mean length of time from the estimated onset of the disease to the diagnosis of dementia [18-20], or they exclusively examined incident dementia cases, and thus were able to report the median time from onset of dementia to institutionalisation [14]. Information about the comparability of reported institutionalisation rates was also provided by data of mean severity of dementia at study entry. For most studies reporting institutionalisation rates, the mean Mini-Mental State Examination score of the demented subsamples ranged between 18 and 22, thus including rather mild to moderate dementia cases.

In figure 1, the empirical findings from the reviewed literature on factors influencing institutionalisation of persons with dementia were integrated in the proposed conceptual framework (see above). Predisposing variables - in our case, sociodemographic and relationship characteristics of the patients and caregivers - were comprehensively analysed in the international literature, and obvious results were usually found. Advanced age and male gender as characteristics of the patients and caregivers were associated with a higher risk of as well as a shorter time to NHP. Results from a recently published meta- 
analysis [49] of NHP of community-dwelling older adults showed that advanced age was also associated with a higher risk for institutionalisation, but with a pooled hazard ratio of 1.03-1.11 compared to a hazard ratio of 1.48$2.00[21,22]$ for demented persons. Furthermore, Gaugler et al. [49] showed comparable results for communitydwelling elderly people as found in this study for demented persons regarding gender (female: hazard ratio of risk: 0.87 vs. 0.81 [31]) and ethnicity (white American: odds ratio of risk: $1.22-2.11$ vs. $1.92-3.30[31,33])$. Ethnic differences in the risk of institutionalisation, particularly between African Americans and white Americans, were observed in the heterogeneous appraisal of dementia-related problem behaviour of the individual with dementia by the African American and white caregivers, with a less stressful appraisal by the African American caregivers [50]. It was suggested that a cultural norm exists in the African American community in which caregiving is an expected experience in adult development $[51,52]$. In the range of predisposing variables, we found a lack of convincing results regarding religiosity and level of education of patients and caregivers. Furthermore, a more profound consideration of the quality of the relationship between patient and caregiver as a modifiable variable is required.

The need variables were divided into primary and secondary stressors. The primary stressors - mainly the dementia-related cognitive, functional and behavioural changes - were widely analysed. A clear association with institutionalisation was found for greater severity of dementia, greater cognitive and functional impairment, and other dementia-related symptoms. It has been supposed that, at a certain point in the course of the disease, it will be too difficult for the caregiver to handle dementia-related symptoms $[35,39]$, especially with stressful behavioural problems such as aggression, depression and hallucinations, leading to the decision of NHP.

The well-examined secondary stressors such as caregiver's burden, life satisfaction and quality of life also led to earlier NHP. Especially the construct 'caregiver's burden' has been considered as a multidimensional response to physical, psychological, emotional, social, and financial (primary) stressors associated with the caregiving experience. But the experience of burden was also mediated by other factors besides the primary stressors, particularly by the caregiver's resources, i.e. the enabling variables, such as personal and social resources as well as community-based care available for the caregivers.

Coping strategies and social support as personal and social resources were marginally analysed. Only Bannis- ter et al. [53] as well as Wells and Over [46] turned to coping strategies of the caregivers, which were also strongly associated with the burden experienced by the caregivers. Results showed a positive effect of active and confrontative coping with needs in the caregiving situation on institutionalisation. Since coping strategies are amenable to modification, further research should focus on coping of informal caregivers aimed at reducing their burden, and with it the risk of institutionalisation.

A positive effect of social support on institutionalisation could only be verified for male patients [41]. Additionally, in that context, family efficiency and the number of children living in the area were examined as aspects of social support. High values of both led to decreased risk of NHP. A closer examination of the construct 'social support' may be beneficial to delay or prevent institutionalisation of demented persons, because an inverse association between a large informal helping network and burden has been shown [54]. In particular, these findings were related to informal sources such as family, neighbours, and friends as well as resulting from instrumental or emotional dimensions of support.

The utilization of community-based services such as adult day services or in-home services showed different effects on institutionalisation. Findings of Gaugler et al. [44] implied that the timing of service use is a potentially important factor often neglected in the analyses. Earlier utilization of CLTC may offer the opportunity for caregivers to accommodate to the range of care demands while receiving assistance. If caregivers waited too long in the disease process to begin service use, especially adult day services, then it functioned more as a transition to NHP than as a form of respite [23], and led to earlier institutionalisation.

Basically, a comparison of study results is restricted by the point of time when studies were conducted. One can assume that it is not unproblematic to compare results from the eighties with the results of today. For example, Cox proportional hazards regression [55], as a multivariate method, has been more frequently applied in studies since 1994, supplying hazard ratios as relative risks. Previously, logistic regression was the method of first choice, providing odds ratios as relative risks. Consideration of further methodological characteristics of the 42 longitudinal studies showed that only 3 studies were based on population-based samples (table 1). The vast majority were based on samples of MC, AD diagnostic centres and CLTC receivers, which limited representativeness and generalisability of results. Moreover, most studies analysed small samples of a few hundred patients and/or 
caregivers, and thus very small subsamples of institutionalised patients, restricting statistical power of multivariate analyses.

The diagnosis of dementia disorders was made inconsistently in the studies. The majority of studies used criteria of international diagnostic systems or NINCDSADRDA criteria. Most others relied on physician's diagnosis without information about the applied diagnostic criteria. These methodological differences limited comparability of results. Examination of NHP risk related to different dementia diagnoses - noted in a few studies on $\mathrm{AD}$ - may yield heterogenous results for several dementia disorders regarding institutionalisation risk and permit diagnosis-based interventions for delay or prevention of institutionalisation in dementia as shown for AD [56].

The present study summarized factors influencing institutionalisation of persons with dementia but did not consider the interactions between these factors. However, interactions between sociodemographic characteristics, primary and secondary stressors and resources as well as between single predictors are another wide research field, and considering that topic would go beyond the scope of the study. Even so, we included potential relations between sociodemographic characteristics, primary and secondary stressors and resources in the conceptual framework derived from findings of the international literature $[7,11]$. Furthermore, we alluded to potential interactions in the discussion section (see above). Additionally, we considered studies which identify predictors exclusively by multivariate analyses to adjust for confounding variables.

The compiled findings on factors influencing institutionalisation of persons with dementia in the present study provided an overview of research activities in this area and differentiated between well-examined and less highlighted factors. This study reveals grey areas where continuous research efforts may facilitate, firstly, the identification of further modifiable factors, and, secondly, the development and application of interventions maintaining persons with dementia in the community for a longer period of time and assuring steps to be taken for a well-timed institutionalisation .

\section{Conclusion}

A comparative analysis of dementia care in OECD countries showed that two policy principles in particular - to remain at home as long as possible and to support caregivers - appear to be universally accepted as desirable [57]. Knowing the circumstances under which dementia patients require admittance to a long-term care institution is important for informing policy. Information about why demented persons and their families face the decision of NHP can help to delay institutionalisation. Few interventions for dementia have unambiguously demonstrated outcomes, but for many this is perhaps a matter of a proper evidence base being built [58]. The need to provide adequate support to carers of individuals with dementia is the second universally accepted policy issue [57]. Financial support by the government for informal caregivers or providing a variety of respite care were two options that can also allow people with dementia to remain in the community as long as possible. The answer to whether there are adequate resources to meet the needs of a growing dementia population depends on a number of factors, including population ageing, changes in options for prevention and treatment, and especially, the availability of informal caregivers.

\section{References}

$>1$ Ferri CP, Prince M, Brayne C, Brodaty H, Fratiglioni L, Ganguli M, et al: Global prevalence of dementia: a Delphi consensus study. Lancet 2005;366:2112-2117.

2 Alzheimer's Association and National Alliance for Caregiving, Families Care: Alzheimer's Caregiving in the United States. Chicago, Alzheimer's Association and Bethesda: National Alliance for Caregiving, 2004.
3 Torti FM Jr, Gwyther LP, Reed SD, Friedman JY, Schulman KA: A multinational review of recent trends and reports in dementia caregiver burden. Alzheimer Dis Assoc Disord 2004;18:99-109.

14 Wimo A, Winblad B, Jonsson L: An estimate of the total worldwide societal costs of dementia in 2005. Alzheimers Dement 2007;3: 81-91.

5 Aguero-Torres H, von Strauss E, Viitanen M, Winblad B, Fratiglioni L: Institutionalization in the elderly: the role of chronic diseases and dementia. Cross-sectional and longitudinal data from a population-based study. J Clin Epidemiol 2001;54:795-801.
6 Goldsmith M: Hearing the Voice of People with Dementia. Opportunities and Obstacles. London, Jessica Kingsley, 1996.

7 Sorensen S, Duberstein P, Gill D, Pinquart M: Dementia care: mental health effects, intervention strategies, and clinical implications. Lancet Neurol 2006;5:961-973.

8 Caron CD, Ducharme F, Griffith J: Deciding on institutionalization for a relative with dementia: the most difficult decision for caregivers. Can J Aging 2006;25:193-205. 
9 Andersen RM: Behavioral Model of Families' Use of Health Services. Research Series No 25. Chicago, Center for Health Administration Studies, University of Chicago, 1968.

10 Andersen RM: Revisiting the behavioral model and access to medical care: does it matter? J Health Soc Behav 1995;36:1-10.

-11 Pearlin LI, Mullan JT, Semple SJ, Skaff MM: Caregiving and the stress process: an overview of concepts and their measures. Gerontologist 1990;30:583-594.

12 Pruchno RA, Michaels JE, Potashnik SL: Predictors of institutionalization among Alzheimer disease victims with caregiving spouses. J Gerontol 1990;45:S259-S266.

13 National Institute for Clinical Excellence: Guideline Development Methods. Information for National Collaborating Centres and Guideline Developers. London, National Institute for Clinical Excellence, 2004.

14 Luck T, Luppa M, Weber S, Matschinger H, Glaesmer H, Koenig H-H, Angermeyer MC, Riedel-Heller S: Time to institutionalization and associated predictors in incident dementia cases - Results of the Leipzig Longitudinal Study of the Aged (LEILA 75+). Neuroepidemiology, submitted.

15 Hébert R, Dubois MF, Wolfson C, Chambers L, Cohen C: Factors associated with longterm institutionalization of older people with dementia: data from the Canadian Study of Health and Aging. J Gerontol A Biol Sci Med Sci 2001;56:M693-M699.

-16 Pot AM, Deeg DJH, Knipscheer CPM: Institutionalization of demented elderly: the role of caregiver characteristics. Int J Geriatr Psychiatry 2001; $16: 273-280$

17 Smith GE, Kokmen E, O’Brien PC: Risk factors for nursing home placement in a population-based dementia cohort. J Am Geriatr Soc 2000;48:519-525.

-18 Smith GE, O’Brien PC, Ivnik RJ, Kokmen E, Tangalos EG: Prospective analysis of risk factors for nursing home placement of dementia patients. Neurology 2001;57:14671473.

19 Severson MA, Smith GE, Tangalos EG, Petersen RC, Kokmen E, Ivnik RJ, et al: Patterns and predictors of institutionalization in community-based dementia patients. J Am Geriatr Soc 1994;42:181-185.

-20 O’Donnell BF, Drachman DA, Barnes HJ, Peterson KE, Swearer JM, Lew RA: Incontinence and troublesome behaviors predict institutionalization in dementia. J Geriatr Psychiatry Neurol 1992;5:45-52.

-21 Andel R, Hyer K, Slack A: Risk factors for nursing home placement in older adults with and without dementia. J Aging Health 2007; 19:213-228

-22 Dorenlot P, Harboun M, Bige V, Henrard JC, Ankri J: Major depression as a risk factor for early institutionalization of dementia patients living in the community. Int J Geriatr Psychiatry 2005;20:471-478.
23 McCann JJ, Hebert LE, Li Y, Wolinsky FD, Gilley DW, Aggarwal NT, et al: The effect of adult day care services on time to nursing home placement in older adults with Alzheimer's disease. Gerontologist 2005;45: 754-763.

24 Stevens A, Owen J, Roth D, Clay O, Bartolucci A, Haley W: Predictors of time to nursing home placement in White and African American individuals with dementia. J Aging Health 2004;16:375-397.

25 Gaugler JE, Kane RL, Kane RA, Clay T, Newcomer R: Caregiving and institutionalization of cognitively impaired older people: utilizing dynamic predictors of change. Gerontologist 2003;43:219-229.

26 Yaffe K, Fox P, Newcomer R, Sands L, Lindquist K, Dane K, et al: Patient and caregiver characteristics and nursing home placement in patients with dementia. JAMA 2002;287:2090-2097.

27 Heyman A, Peterson B, Fillenbaum G, Pieper C: Predictors of time to institutionalization of patients with Alzheimer's disease: the CERAD experience, part XVII. Neurology 1997;48:1304-1309.

28 Gilley DW, Bienias JL, Wilson RS, Bennet DA, Beck TL, Evans DA: Influence of behavioral symptoms on rates of institutionalization for persons with Alzheimer's disease. Psychol Med 2004;34:1129-1135.

29 Miller SC, Prohaska TR, Furner SE: Nursing home admission for African Americans with Alzheimer's disease. J Gerontol A Biol Sci Med Sci 1999;54:M365-M369.

30 Banerjee S, Murray J, Foley B, Atkins L, Schneider J, Mann A: Predictors of institutionalisation in people with dementia. J Neurol Neurosurg Psychiatry 2003;74:13151316.

-31 Gaugler JE, Kane RL, Kane RA, Newcomer R: Unmet care needs and key outcomes in dementia. J Am Geriatr Soc 2005;53:20982105.

32 de Vugt ME, Stevens F, Aalten P, Lousberg R, Jaspers N, Verhey FR: A prospective study of the effects of behavioral symptoms on the institutionalization of patients with dementia. Int Psychogeriatr 2005;17:577-589.

33 Scott WK, Edwards KB, Davis DR, Cornman $\mathrm{CB}$, Macera CA: Risk of institutionalization among community long-term care clients with dementia. Gerontologist 1997;37:4651.

34 Spruytte N, Van Audenhove C, Lammertyn F: Predictors of institutionalization of cognitively-impaired elderly cared for by their relatives. Int J Geriatr Psychiatry 2001;16:11191128 .

35 Coehlo DP, Hooker K, Bowman S: Institutional placement of persons with dementia. J Fam Nurs 2007;13:253-277.

36 Gaugler JE, Kane RL, Kane RA, Newcomer $\mathrm{R}$ : Predictors of institutionalization in Latinos with dementia. J Cross Cult Gerontol 2006;21:139-155.
37 Miller SC, Prohaska TR, Furner SE, Freels S, Brody JA, Levy PS: Time to nursing home admission for persons with Alzheimer's disease: the effect of health care system characteristics. J Gerontol B Psychol Sci Soc Sci 1998;53:S341-S353.

38 Gaugler JE, Leach CR, Clay T, Newcomer RC: Predictors of nursing home placement in African Americans with dementia. J Am Geriatr Soc 2004;52:445-452.

-39 Buhr GT, Kuchibhatla M, Clipp EC: Caregivers' reasons for nursing home placement: clues for improving discussions with families prior to the transition. Gerontologist 2006;46:52-61.

40 Haupt M, Kurz A: Predictors of nursing home placement in patients with Alzheimer's disease. Int J Geriatr Psychiatry 1993; 8:741-746.

41 Young RF: Nursing home admission of female Alzheimer's patients: family care aspects. Womens Health Issues 2003;13:2-7.

42 Zarit SH, Reever KE, Bach-Peterson J: Relatives of the impaired elderly: correlates of feelings of burden. Gerontologist 1980;20: 649-655.

43 Argimon JM, Limon E, Vila J, Cabezas C: Health-related quality-of-life of care-givers as a predictor of nursing-home placement of patients with dementia. Alzheimer Dis Assoc Disord 2005;19:41-44.

-44 Gaugler JE, Kane RL, Kane RA, Newcomer R: Early community-based service utilization and its effects on institutionalization in dementia caregiving. Gerontologist 2005;45: 177-185.

45 Fisher L, Lieberman MA: A longitudinal study of predictors of nursing home placement for patients with dementia: the contribution of family characteristics. Gerontologist 1999;39:677-686.

46 Wells Y, Over R: Institutional placement of a dementing spouse: the influence of appraisal, coping strategies, and social support. Aust J Psychol 1998;50:100-105.

47 Bharucha AJ, Pandav R, Shen C, Dodge HH, Ganguli M: Predictors of nursing facility admission: a 12 -year epidemiological study in the United States. J Am Geriatr Soc 2004;52: 434-439.

48 Eaker ED, Vierkant RA, Mickel SF: Predictors of nursing home admission and/or death in incident Alzheimer's disease and other dementia cases compared to controls: a population-based study. J Clin Epidemiol 2002;55: 462-468.

49 Gaugler JE, Duval S, Anderson KA, Kane RL: Predicting nursing home admission in the US: a meta-analysis. BMC Geriatr 2007; $7: 13$.

50 Haley WE, Roth DL, Coleton MI, Ford GR, West CA, Collins RP, Isobe TL: Appraisal, coping, and social support as mediators of well-being in black and white family caregivers of patients with Alzheimer's disease. J Consult Clin Psychol 1996;64:121-129. 
51 Rosenthal CJ: Family supports in later life: does ethnicity make a difference? Gerontologist 1986;26:19-24.

52 Dilsworth-Anderson P, Williams SW, Cooper T: The context of experiencing emotional distress among family caregivers to elderly African-Americans. Fam Relat 1999;48: 391-396.

-53 Bannister C, Ballard C, Lana M, Fairbairn A, Wilcock G: Placement of dementia sufferers in residential and nursing home care. Age Ageing 1998;27:189-193.

54 Vrabec NJ: Literature review of social support and caregiver burden, 1980 to 1995 . Image J Nurs Sch 1997;29:383-388.

55 Cox DR: Regression models and life tables. J R Stat Soc Ser B 1972;34:187-220.

-56 Cummings JL, Knopman D: Advances in the treatment of behavioral disturbances in Alzheimer's disease. Neurology 1999;53: 899-901.
57 Moise P, Schwarzinger M, Um M, the Dementia Experts' Group: Dementia Care in 9 OECD Countries: A Comparative Analysis. Paris, OECD Health Working Papers, 2004.

58 Sorensen S, Duberstein P, Gill D, Pinquart M: Dementia care: mental health effects, intervention strategies, and clinical implications. Lancet Neurol 2006;5:961-973.

59 Soto ME, Andrieu S, Gillette-Guyonnet S, Cantet C, Nourhashemi F, Vellas B: Risk factors for functional decline and institutionalisation among community-dwelling older adults with mild to severe Alzheimer's disease: one year of follow-up. Age Ageing 2006 35:308-310.

60 Gaugler JE, Kane RL, Kane RA, Clay T, Newcomer RC: The effects of duration of caregiving on institutionalization. Gerontologist 2005;45:78-89.

61 Andrieu S, Reynish W, Nourhashemi F, Ous set PJ, Grandjean H, Grand A, et al: Nutritional risk factors for institutional placement in Alzheimer's disease after one year followup. J Nutr Health Aging 2001;5:113-117.
62 Hope T, Keene J, Gedling K, Fairburn CG, Jacoby R: Predictors of institutionalization for people with dementia living at home with a carer. Int J Geriatr Psychiatry 1998;13:682690.

63 Wright LK: Alzheimer's disease afflicted spouses who remain at home: can human dialectics explain the findings? Soc Sci Med 1994;38:1037-1046.

64 Lieberman MA, Kramer JH: Factors affecting decisions to institutionalize demented elderly. Gerontologist 1991;31:371-374.

65 Knopman DS, Kitto J, Deinard S, Heiring J: Longitudinal study of death and institutionalization in patients with primary degenerative dementia. J Am Geriatr Soc 1988;36: 108-112.

66 Colerick EJ, George LK: Predictors of institutionalization among caregivers of patients with Alzheimer's disease. J Am Geriatr Soc 1986;34:493-498. 